\title{
Black hole phase transitions in Hořava-Lifshitz gravity
}

\author{
Qiao-Jun Cao * Yi-Xin Chen $t$ and Kai-Nan Shad \\ Zhejiang Institute of Modern Physics, \\ Zhejiang University, Hangzhou, 310027, China
}

\begin{abstract}
We study black hole phase transitions in (deformed) Hořava-Lifshitz (H-L) gravity, including the charged/uncharged topological black holes and KS black hole. Stability analysis and state space geometry are both used. We find interesting phase structures in these black holes, some of the properties are never observed in Einstein gravity. Particularly, the stability properties of black holes in H-L gravity with small radius change dramatically, which can be considered as a leak of information about the small scale behavior of spacetime. A new black hole local phase transition in H-L gravity which cannot be revealed by thermodynamical metrics has been found. There is an infinite discontinuity at the specific heat curve for charged black hole in H-L gravity with hyperbolic event horizon. However, this discontinuity does not have a corresponding curvature singularity of thermodynamical metrics. Our results may provide new insights towards a better understanding of the H-L gravity, as well as black hole thermodynamics.
\end{abstract}

\footnotetext{
*Electronic address: caoqiaojun@gmail.com

${ }^{\dagger}$ Electronic address: yxchen@zimp.zju.edu.cn

${ }^{\ddagger}$ Electronic address: shaokn@gmail.com
} 


\section{INTRODUCTION}

Due to the celebrated work of Bekenstein[1] and Hawking[2] on black hole physics, it is widely accepted that black holes are thermodynamic systems. The four laws of black hole thermodynamics[3] have been put on solid fundamentals. It is also believed that some kinds of unknown microscopic degrees of freedom are coming into play since every black hole has a temperature proportional to the surface gravity on the black hole event horizon and an entropy proportional to the area of event horizon.

Phase transition is an important phenomenon in ordinary thermodynamics. It is therefore natural to ask whether there are some phase transitions in black hole thermodynamics. The pioneering work of Hawking and Page proved that there is a global phase transition between thermal AdS state and AdS black hole in four dimensions as the temperature changes [4]. It means that thermal radiation in AdS space becomes unstable at a certain temperature, and eventually collapses to form a black hole. However, physical difference in the microscopic structure in different phases is still unclear. Witten reconsidered HawkingPage phase transition in the spirit of AdS/CFT correspondence, and interpreted it as a transition between low-temperature confining and high temperature deconfining phase in the dual field theory[5]. The research of black hole phase transition has been extended and indicate that there may exist different phase transitions in various circumstance [6-19]. The signal of a phase transition is typically characterized by the sign change of a capacity, such as the specific heat, charge capacitance or moment of inertia, by going through either zero or infinity. Davies argued that the points that the specific heat with fixed charge and/or angular momentum passing from negative to positive values through an infinite discontinuity are commonly associated with phase transitions[20, 21]. These divergences in the phase space are denoted as Davies curves now. We should also note that this type of thermodynamical phase transition is based on the local stability analyses and is generally different from the global Hawking-Page phase transition. In this paper, we shall keep an eye to distinguish the local and global stabilities of black hole thermodynamic ensembles.

The study of phase transition has been extended in various directions, e.g., to black holes with non-spherical event horizon topologies[22, 23], to black holes including more conserved quantities such as charges and angular momenta [6-10] or to higher dimensional black holes[10]. The research can also be generalized to gravity theories with high derivatives 
of curvature terms, such as Gauss-Bonnet gravity [11, 12, 18], Born-Infeld gravity [15-17] and Lovelock gravity etc[13, 14, 19]. In these cases, phase transitions and critical phenomena are more complicated.

Weinhold [24] and Ruppeiner [25, 26] introduced thermodynamical metrics to study thermodynamic systems. A thermodynamic system is described by some thermodynamical quantities, and these quantities are all related to each other. Weinhold took internal energy $M$ as a function of entropy $S$ and other extensive variables $N^{a}$ which are necessary to describe the thermodynamic system. Denoting the collection of all the other thermodynamical quantities except $M$ as $\left\{S^{i}\right\} \equiv\left\{S, N^{a}\right\}$, then $M=f\left(S^{i}\right)$. He constructed a metric $d s^{2}(M)=h_{i j} d S^{i} d S^{j}$, where $h_{i j}=\partial^{2} f\left(S^{i}\right) /\left(\partial S^{i} \partial S^{j}\right)$ is the Hessian matrix of $f\left(S^{i}\right)$. In the similar way, Ruppeiner later introduced a new metric $d s^{2}(S)$ by using the entropy as a generating function depending on other extensive variables[25]. Remarkably, the curvature of Ruppeiner metric could be related to interactions in the underlying statistical system and scaled as the correlation volume. It is divergent at critical points of second order phase transitions. It has been proved that Weinhold and Ruppeiner metrics are conformally equivalent to the inverse of the temperature as the conformal factor. There are amounts of works in discussing the relationship of black hole phase transitions and Ruppeiner/Weinhold metrics [27 30]. The Ruppeiner and Weinhold metrics indeed reveal some signals of black hole phase transitions, but sometimes they fail to work to the Davies curve[29].

Inspired by Weinhold and Ruppeiner, a framework was proposed to identify all of the phase transition points in black hole thermodynamics[31]. The authors of [31] introduced metrics based on all thermodynamical potentials generated by Legendre transformations of the black hole energy or entropy, and found that the collection of all phase transition points are in one-to-one correspondence to the collection of curvature singularities of those thermodynamical metrics. This work clarified that the divergences in the associated set of thermodynamical geometries can reveal the threshold points of all capacities completely. A natural question is "what is range of application of the correspondence between curvature singularities of the generalized thermodynamical metrics and phase transition points in black hole thermodynamics?" or "Is the correspondence valid for all thermodynamical systems? e.g. black hole solutions among different gravity theories such as Born-Infeld gravity and Lovelock gravity etc." Though various black holes with spherical topology of horizon and black ring in five dimensions with single angular momentum were investigated 
in [31], it is far from a complete proof of the correspondence between curvature singularities of thermodynamical metrics and phase transition signals. It will be very interesting to investigate some black holes in other gravity theories or with non-spherical topology of horizon that may provide counter-examples to this correspondence, which may help to identify the correspondence's range of application.

Recently, a new theory of gravity at a Lifshitz point was proposed by Hořava, which may be regarded as a UV complete candidate for general relativity[32-34]. The Hořava-Lifshitz (H-L) theory has been intensively investigated. Some kind of black hole solutions for H-L gravity have been found [35-38]. The thermodynamic analysis of H-L gravity black holes is a nontrivial task and has been done in [36, 39]. Nevertheless, the charged black hole in H-L gravity has not been studied from the thermodynamic perspective. Moreover, in [40] and [41], the authors examined the thermodynamical geometry of the topological black hole in H-L theory and KS black hole, respectively. Their papers only constructed the Ruppeiner and Weinhold metrics. However, the new metric based on free energy which was introduced in [31] should be included for a full analysis. These prompt us to study the stability of black holes and their phase transitions in H-L gravity. It is also necessary to construct their whole set of thermodynamical metrics and calculate the associated Ricci scalars, and to examine the correspondence mentioned in the previous paragraph.

In this paper, we investigate possible black hole phase transitions in H-L gravity carefully, including the charged/uncharged topological black hole in H-L gravity and KS black hole. We discuss stability of these black holes as well as the phase transition signals using both analytical and graphical techniques, especially in the ensemble with fixed charge parameters. The phase structures are vivid in these kinds of black holes in H-L gravity, which are very different from the black holes in Einstein gravity. Thermodynamic properties of the topological black holes in H-L gravity are reexamined, and new observations have been made. We analyze the specific case with dynamical coupling constant $\lambda=1$ carefully, for black holes with hyperboloid topology. It turns out that there is a phase transition going from small to large black holes at a critical temperature, of first order. For charged black hole in H-L gravity, in the ensemble with fixed charge parameters, we found that the black holes with sphere or flat topology have no phase transition in the canonical ensemble with fixed charge, since the specific heat with fixed charge $C_{Q}$ is positive definite. However, the thermodynamic behavor of RN AdS black holes is different, there can be three branches of 
RN AdS black holes with a small fixed charge, and the middle radius branch is unstable, with the temperature increases, small black holes can phase transition to large black holes[7, 8]. The charged black hole in H-L gravity with hyperboloid topology is extremely interesting. There are three branches of black holes. The branch with middle radius is local unstable while the small and large branches are local stable. This signifies a local phase transition at the point with divergent specific heat, but there is no global phase transition here since the branch with largest radius always have minimum free energy at all temperatures. For KS black hole, it is obvious to see that the high derivative terms in the H-L gravity action play an important role. Their influences on the KS black hole thermodynamic properties reduced to a charge like parameter, and make it thermodynamically behaving like a RN black hole. Compared to the Schwarzschild black hole it has a new stable phase with a small radii, and phase transition comes up.

The thermodynamical metrics of black holes in H-L gravity are also constructed, and the corresponding Ricci scalars are calculated for the purpose of investigating their curvature singularities. We found that all the cases we discussed in this paper are almost consistent with the framework proposed in [31], except that there is a infinite discontinuity at the specific heat curve for charged black hole with hyperbolic event horizon in H-L gravity, while this discontinuity does not has a corresponding curvature singularities of thermodynamical metrics. This is a probable counter-example to the correspondence between local phase transition points and curvature divergences of thermodynamical geometries. This also indicate that the thermodynamical metrics can not reveal all of the local phase transition signals in H-L gravity. The violation of this correspondence in charged black holes with hyperbolic event horizon in H-L gravity may be related to the non-spherical topology of it's event horizon. The ultraviolet behavior of spacetime in $\mathrm{H}-\mathrm{L}$ gravity deserves as the reason for all of those strange properties in black hole thermodynamics. Our results may provide new insights towards a better understanding of the Hořava-Lifshitz gravity, as well as black hole thermodynamics.

This paper is organized as follows. In Section [I], we focus on the thermodynamic properties of topological black holes in H-L gravity, and recall the stability analyses by using their heat capacity and free energy. In Section III, we consider the charged topological black holes in H-L gravity. Their stability and the phase transition signals are examined carefully. Both the specific heats $C_{Q}$ and $C_{\Phi}$, and charge capacitances $\tilde{C}_{T}$ and $\tilde{C}_{S}$ are calculated out. The 
threshold points where these quantities change sign through zero or infinite discontinuity are compared with the divergent points of the Ricci scalar curvatures for Weinhold metric $d s^{2}(M)$, Ruppeiner metric $d s^{2}(S)$ and the free-energy metric $d s^{2}(F)$. We found that not all threshold points in the four $C$ 's match precisely to those singularities in the three metrics. In Section IV, the thermodynamical properties of KS black holes were investigated. It thermodynamically behaves like a RN black hole, and phase transition comes up. We see that all possible phase transitions found from the four $C$ 's correspond to curvature singularities of certain thermodynamical metrics, which are consistent very well to the framework proposed by [31]. Section $\nabla$ is for summary and discussions. In the appendix, we examine the thermodynamical properties of KS black holes by defining the mass and the entropy in an alternative way.

\section{TOPOLOGICAL BLACK HOLE IN HOŘAVA-LIFSHITZ GRAVITY}

Thermodynamic properties of the topological black holes in H-L gravity have been examined in [39, 40]. We review it briefly in this section and make some new observations. For general dynamical coupling constant $\lambda$ in H-L gravity action, the solution of the topological black holes in H-L gravity was first obtained in [36]. The metric can be written as

$$
d s^{2}=-\tilde{N}^{2} f(r) d t^{2}+\frac{d r^{2}}{f(r)}+r^{2} d \Omega_{k}^{2},
$$

where $d \Omega_{k}^{2}$ denotes the line element for two-dimensional Einstein space with constant scalar curvature $2 k$. We can take (without loss of generality) $k=0, \pm 1$ for plane, sphere or hyperboloid 2 -space respectively. The function $f(r)$ is given by

$$
f(r)=k-\Lambda r^{2}-\alpha r^{s} \quad, \quad \tilde{N}=\gamma r^{1-2 s},
$$

where

$$
s=\frac{2 \lambda \pm \sqrt{2(3 \lambda-1)}}{\lambda-1},
$$

and $\alpha$ and $\gamma$ are both integration constants. The black hole horizon is at $r=r_{+}$, which is defined by the largest real positive root of $f(r)=0$. There are two branches of solution according to the sign in (3). It is reasonable to choose the negative branch since the physical meaning of the positive branch is not very clear [39]. The range of $s$ is $(-1,2)$ for the negative branch when $\lambda>1 / 3$. 
It should be noticed that the asymptotically behavior of those solutions are complicated. They are neither asymptotically flat nor asymptotically AdS, so we have to use the canonical Hamiltonian method to define their mass. Defining $l^{2}=-\frac{1}{\Lambda}$, the thermodynamical quantities are collected as[39]

$$
\begin{aligned}
T & =\frac{\gamma}{8 \pi r_{+}}\left(-\Lambda r_{+}^{2}(2-s)-s k\right)=\frac{\gamma}{4 \pi r_{+}^{2 s}}\left[\left(\frac{r_{+}}{l}\right)^{2}(2-s)-k s\right], \\
S & =\frac{\pi \kappa^{2} \mu^{2} \Omega_{k}}{\sqrt{2(3 \lambda-1)}}\left[k \ln \left(\sqrt{-\Lambda} r_{+}\right)+\frac{1}{2}\left(\sqrt{-\Lambda} r_{+}\right)^{2}\right]+S_{0} \\
M & =\frac{\sqrt{2} \kappa^{2} \mu^{2} \Omega_{k}}{16 \sqrt{3 \lambda-1}} \gamma \alpha^{2}=\frac{\sqrt{2} \kappa^{2} \mu^{2} \Omega_{k} \gamma}{16 \sqrt{3 \lambda-1}} \frac{\left(k-\Lambda r_{+}\right)^{2}}{r_{+}^{2 s}}
\end{aligned}
$$

Using

$$
c=\frac{\kappa^{2} \mu}{4} \sqrt{\frac{\Lambda}{1-3 \lambda}}=\left(\frac{2-s}{1+s}\right)\left(\frac{\kappa^{2} \mu}{4 \sqrt{2} l}\right)
$$

one may rewrite

$$
\begin{gathered}
M=\frac{c^{3}}{16 \pi G}\left(\frac{1+s}{2-s}\right)\left(\gamma \Omega_{k} l^{2-2 s}\right)\left[\frac{k+\left(\frac{r_{+}}{l}\right)^{2}}{\left(\frac{r_{+}}{l}\right)^{s}}\right]^{2}, \\
S=\frac{c^{3}}{4 G}\left(\frac{1+s}{2-s}\right)\left(\Omega_{k} l^{2}\right)\left[k \ln \left(\frac{r_{+}}{l}\right)^{2}+\left(\frac{r_{+}}{l}\right)^{2}\right]+S_{0} .
\end{gathered}
$$

It is easy to confirm that the above thermodynamical quantities satisfy the first law of thermodynamics $d M=T d S$.

Two points should be noticed here which did not appear in the analyses of Schwarzschild AdS black holes in [4], viz., the expression of mass (8) is not monotonous increasing with $r_{+}$ when $k= \pm 1$, and there is a logarithmic term in the entropy expression unless $k=0$, one cannot fix the integration constant $S_{0}$ here. The sign of the specific heat for the topological black holes in H-L gravity is different from the sign of local slope of the $T\left(r_{+}\right)$curve. We should be careful about them in the following discussion.

It is instructive to plot the temperature as a function of horizon radius for the topological black holes in H-L gravity (see Figure 1). In these figures, we have taken the parameters $c=l=G=\gamma=\Omega_{k}=1$ and $s=\frac{1}{2}(\lambda=1)$. As can be seen from Figure 1c, the temperature for $k=-1$ has a minimum, which locates at $r_{+}=\frac{s}{\sqrt{2-3 s+s^{2}}}$. Above the minimum temperature, there are two black holes with different radius associated with the same temperature. Below this temperature, no black hole exists. Those facts may indicate that there is a phase transition at this point. Checking the expression of temperature Eq.(44), 
one can see that the minimum temperature only exists for $0<s<1$. Figure 2 depicts the phase structure for topological black holes with $k=-1$. As shown in Figure 2, those black holes only exists above a minimum temperature. This is qualitatively similar to the familiar case of the Schwarzschild AdS black holes studied in [4]. It should be emphasized that this is not all the story of the black holes discussed here. This is because the expressions of mass and entropy are not monotonous increase with horizon radius when $k=-1$, and we cannot conclude that the black hole with smaller radii is unstable while the larger one is stable. To be more precise, the sign of the specific heat $C=\frac{\partial M}{\partial T}=T \frac{\partial S}{\partial T}$, which determines the local stability of a thermodynamic system, cannot be inferred from the local slope of the $T\left(r_{+}\right)$curve for the topological black holes in H-L gravity. This is very different from the Hawking-Page transition. The local stability properties of those black holes should be analyzed by using the specific expression of specific heat.

It is also worth to mention another interesting issue that the temperature behaviors of the topological black holes in $\mathrm{H}-\mathrm{L}$ gravity with $k=1,0$ and -1 are very similar to the black holes with $k=-1,0$ and 1 in Einstein's general relativity, respectively, which has been first emphasized in [36]. 


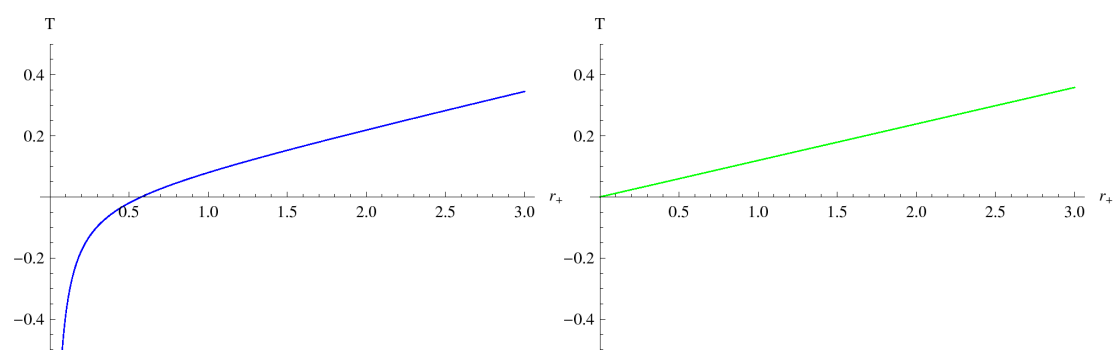

(a) $k=1$

(b) $k=0$

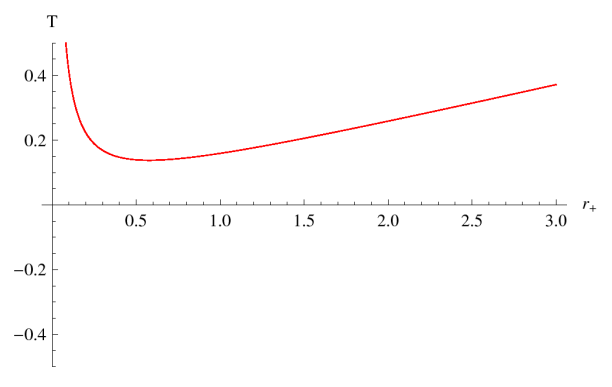

(c) $k=-1$

Figure 1: Temperature for topological black holes vs. horizon radius $r_{+}$, with $c=l=G=$ $\gamma=\Omega_{k}=1$ and $s=\frac{1}{2}$. For $k=1$, the negative temperature region $r_{+}<\frac{1}{\sqrt{3}}$ is unphysical, only the positive temperature region have physical significance, the zero temperature point is where the black hole is extremal. In the case of $k=-1$, the behavior of the temperature is similar to Schwarzschild AdS black hole. (see text for discussion of this case.) 


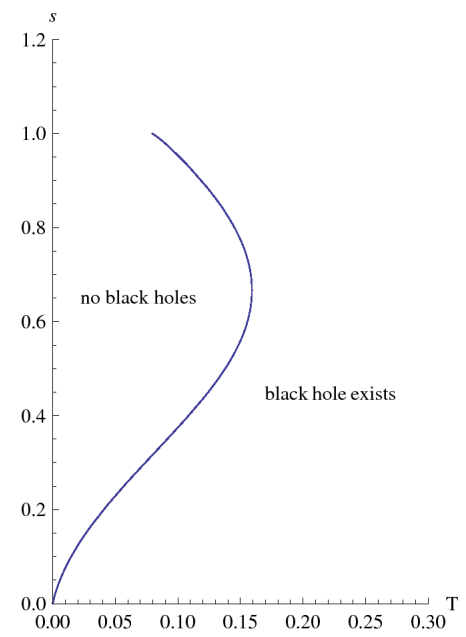

Figure 2: Phase structure for topological black holes with $k=-1$ and $0<s<1$. Black holes only exists in the right side of the curve of minimum temperature, i.e. above a certain temperature.

To study the local stability of topological black holes in H-L gravity, we need to calculate the heat capacity,

$$
C_{\lambda}=\left.\frac{\partial M}{\partial T}\right|_{\lambda}=\frac{c^{3} l^{-2 s} r^{2 s}\left(\frac{r}{l}\right)^{-2 s}\left(k l^{2}+r^{2}\right)(1+s)\left(r^{2}(-2+s)+k l^{2} s\right) \Omega_{k}}{4 G(-2+s)\left(r^{2}(-2+s)(-1+s)+k l^{2} s^{2}\right)} .
$$

If we set $\lambda=1$, it reduces to the expression discussed in [36]. We show the special case of $s=\frac{1}{2}(\lambda=1)$ in Figure 3 . 


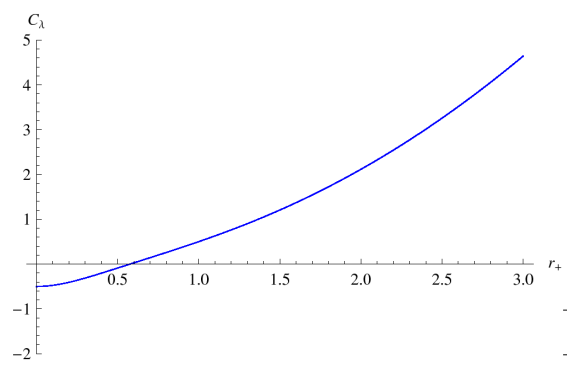

(a) $k=1$

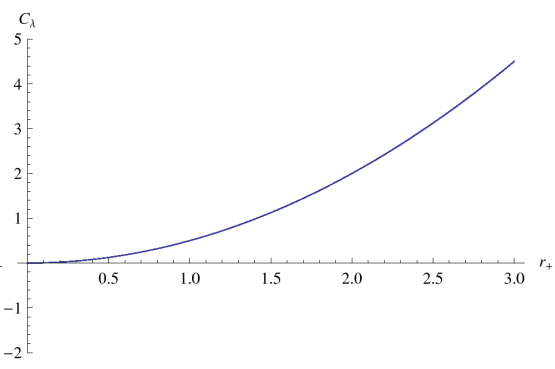

(b) $k=0$

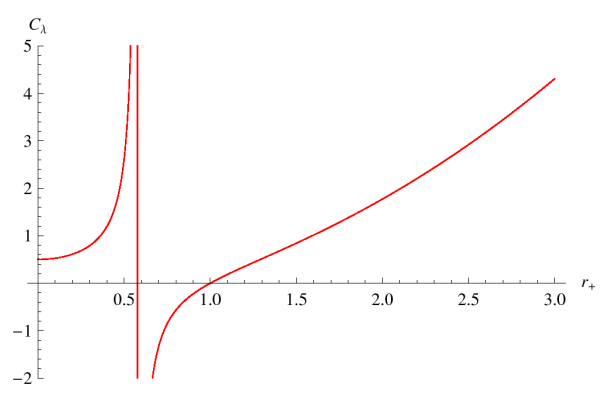

(c) $k=-1$

Figure 3: Heat capacity for topological black holes $v s$. horizon radii $r_{+}$, with $c=l=G=$ $\gamma=\Omega_{k}=1$ and $s=\frac{1}{2}$. For $k=1$, the heat capacity changes from negative values to positive values through a zero point has nothing to do with phase transition, the negative region $r_{+}<\frac{1}{\sqrt{3}}$ is unphysical, and the zero point is associated with a extremal black hole. The divergence in the last graph is associate with the minimum temperature in Figure 1c, There are three branches of black holes, the middle branch is local unstable with negative values of heat capacity while the small and large branches are local stable.

From Figure 3c, we see that there is a divergent point of the heat capacity for $k=-1$. The divergent point, $r_{+}=\frac{s}{\sqrt{s^{2}-3 s+2}}$, coincides with the value of $r_{+}$for minimum temperature. Across this point, the local stability of black hole changes. In order to study the phase structure and global stability, we must observe the free energy, it can be obtained as follows: $F=M-T S=-\frac{c^{3}(1+s) \gamma\left(r^{-2 s}\left(k l^{2}+r^{2}\right)^{2}+r^{-2 s}\left(r^{2}(-2+s)+k l^{2} s\right)\left(r^{2}+k l^{2} \ln \left(\frac{r^{2}}{l^{2}}\right)\right)\right) \Omega_{k}}{16 G l^{2} \pi(-2+s)}$.

To see its properties, we plot the free energy for $s=\frac{1}{2}(\lambda=1)$ in Figure 4 . The maximum point of the free energy for $k=-1$ in Figure $4 \mathrm{c}$ is $r_{+}=\frac{s}{\sqrt{2-3 s+s^{2}}}$, which coincides with with the point for the minimum temperature and divergent heat capacity. 


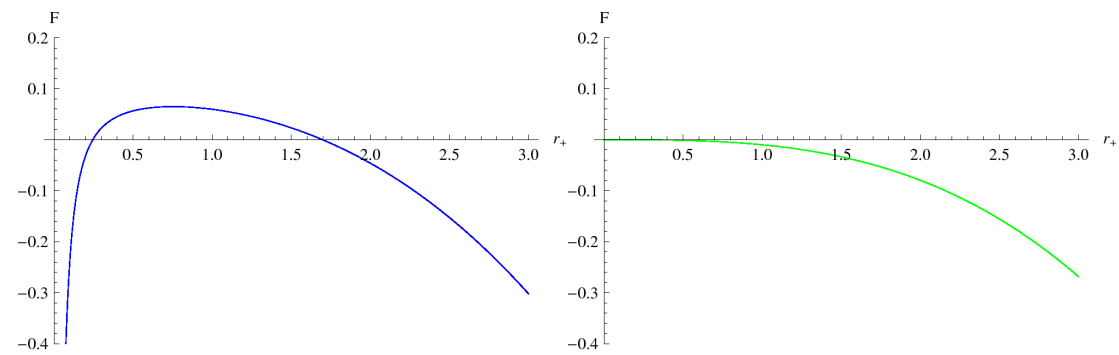

(a) $k=1$

(b) $k=0$

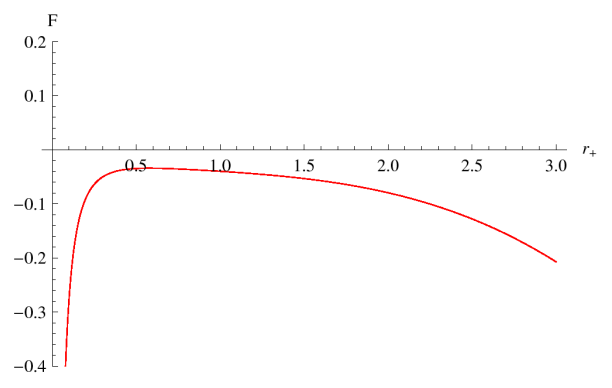

(c) $k=-1$

Figure 4: Free energy for topological black holes vs. horizon radii $r_{+}$, with $c=l=G=\gamma=$ $\Omega_{k}=1$ and $s=\frac{1}{2}$. 


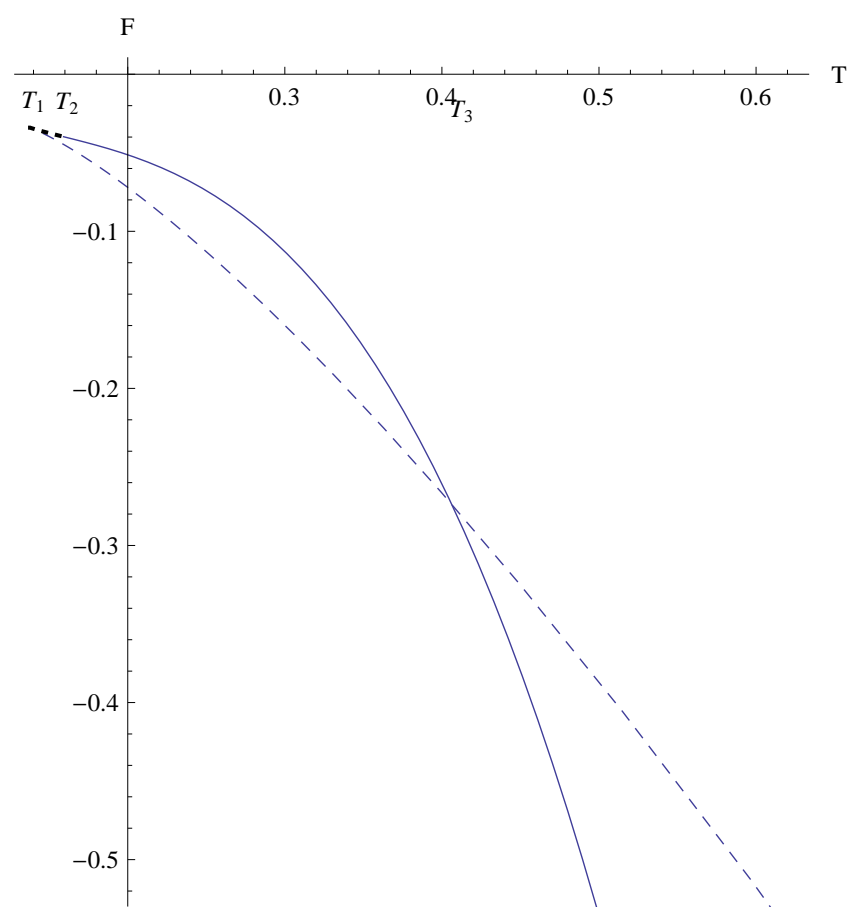

Figure 5: Free energy for topological black holes with $k=-1$ vs. temperature, with $c=l=G=\gamma=\Omega_{k}=1$ and $s=\frac{1}{2}$. The thick dotted curve is for the middle branch, while the dashed and solid curves are associated with the small and large branches, respectively. Note that the origin coordinates are $(0.2,0)$.

The free energy as a function of temperature for $k=-1$ is showed in Figure 5. There is no black holes below a certain temperature $T_{1}\left(T_{1}=\frac{\sqrt{3}}{4 \pi}\right.$ in the plot). Above $T_{1}$, two black holes can exist and the small branch is the more stable than the middle branch. As the temperature increasing, at temperature $T_{2}\left(T_{2}=\frac{1}{2 \pi}\right.$ in the plot), the large branch take the place of middle branch, but the free energy of the small branch is still more negative than that of the large branch. As the temperature increases further, the small and large branches have the same free energy at $T_{3}$ ( $T_{3}$ is about 0.406 in the plot), above this temperature, the large branch takes over the physics. This signifies a phase transition goes from small to large $r_{+}$black holes. At $T_{3}$, the free energy is continuous, but its first derivative is discontinuous. This is a typical first order phase transition .

The above results can be interpreted as follow. For our specific choice $s=\frac{1}{2}$, the thermodynamic properties of the topological black holes in H-L gravity with different horizon topologies are very different from each other. For $k=1$, the free energy decreases steadily in the physical region. Any non-extremal black holes have positive heat capacity, and the 
black holes are stable. Thermodynamical behavior of the $k=0$ case is just like the $k=1$ case. The story is very different for $k=-1$ case. It can be inform from Figure $3 \mathrm{c}$ that there are three branches of black holes. The middle branch is unstable while the small and large branches are stable. The branch with the smallest radii is new in H-L gravity, and does not appear in the Schwarzschild AdS black hole. Also notice that the middle branch has the highest free energy, and is relatively unstable.

We have found interesting phase structures in the topological black holes in H-L gravity, some of the properties are never observed in Einstein gravity. The H-L gravity is regarded as a UV complete candidate for general relativity, and the ultraviolet behavior of spacetime changed dramatically, maybe, this is why there is a branch of stable black hole with a small radii appear in the $k=-1$ case. Pursuing its deep reason goes beyond the scope of this paper, and we will leave settling of this interesting issue to a future date. For general dynamical coupling constant $\lambda$, the heat capacity for three cases with different horizon topologies can all have positive and negative values, which means that there always exist locally thermodynamically stable phases and unstable phases in suitable parameter regimes. This indicate phase transitions at certain threshold points. Relevant results can be found in [39].

In [40], the authors obtained the Ruppeiner metric for this kind of black holes by taking $\lambda$ and $M$ as variables. Weinhold metric was also calculated there. However, in Hořava-Lifshitz gravity, $\lambda$ represents a dynamical coupling constant, susceptible to quantum corrections, it is not a conserved charge, a convincing reason is needed for why it can be taken as a variable in constructing thermodynamic metrics. Taking this argument into account, one see that the thermodynamical metric of the uncharged black hole is one dimensional, so we do not study the various properties of the scalar curvatures.

\section{CHARGED BLACK HOLE IN HOŘAVA-LIFSHITZ GRAVITY}

Phase transitions in charged black holes such as Reissner-Nordström black holes have been studied in $[6] 8,20,42]$.In this section we extend the discussion of phase transitions to charged black holes in H-L gravity. For simplicity and clearness we set $\lambda=1$. The charged topological black hole solution in H-L gravity for $\lambda=1$ has been given in [36], the metric is 


$$
\begin{aligned}
& d s^{2}=-\tilde{N}(r)^{2} f(r) d t^{2}+\frac{d r^{2}}{f(r)}+r^{2} d \Omega_{k}^{2} \\
& f(r)=k+x^{2}-\sqrt{c_{0} x-\frac{q^{2}}{2}}, \quad x=\sqrt{-\Lambda} r
\end{aligned}
$$

where $\tilde{N}=N_{0}$ could be set to one. The event horizon radius is determined by $x_{+}$(note $x_{+}$ is not the horizon radius), which is the largest positive root of $f(x)=0$. Denoting $l^{2}=-\frac{1}{\Lambda}$ as well, the thermodynamical quantities computed in [36] are

$$
\begin{aligned}
T & =\frac{6 x_{+}^{4}+4 k x_{+}^{2}-2 k^{2}-q^{2}}{16 k l^{2} \pi x_{+}+16 l^{2} \pi x_{+}^{3}}, \\
S & =\frac{\pi \kappa^{2} \mu^{2} \Omega_{k}}{4}\left(x_{+}^{2}+2 k \ln \left(x_{+}\right)\right)+S_{0}, \\
\Phi & =\frac{q}{x_{+}}+\Phi_{0}, \\
Q & =\frac{\kappa^{2} \mu^{2} \Omega_{k}}{16 l^{2}} q, \\
M & =\frac{\kappa^{2} \mu^{2} \Omega_{k}}{16 l^{2}} c_{0},
\end{aligned}
$$

where $c_{0}=\frac{2 k^{2}+q^{2}+4 k x_{+}^{2}+2 x_{+}^{4}}{2 x_{+}}$. As we can see, the mass of the charged topological black hole in $\mathrm{H}-\mathrm{L}$ gravity is also not increasing monotonously with $x_{+}$. It is more complicate than the uncharged one, since the charge parameter $q$ comes into play. The charge parameter $q$ does not appear explicitly in the expression of entropy $S$. It is consistent with the fact that black hole entropy is a function of horizon geometry. It is easy to verify that the first law of thermodynamic $d M=T d S+\Phi d Q$ is satisfied. 


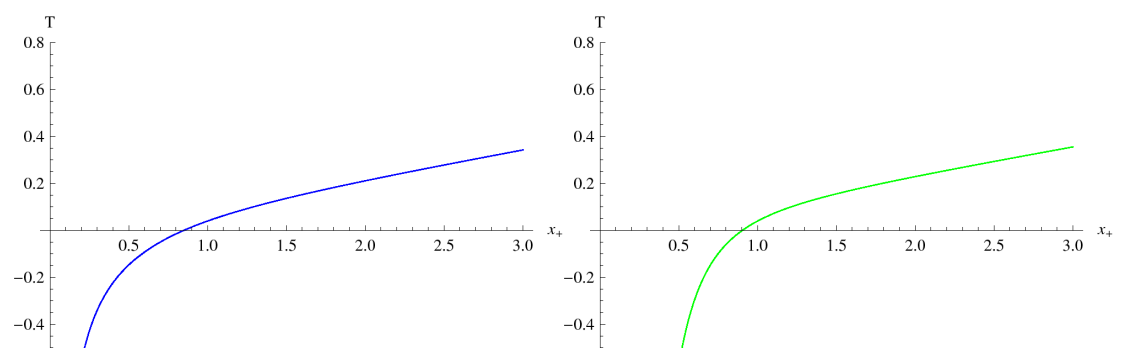

(a) $k=1$

(b) $k=0$

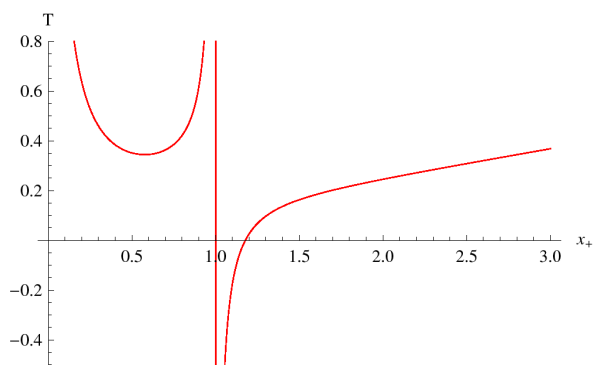

(c) $k=-1$

Figure 6: Temperature of charged topological black holes vs. $x_{+}$, the values $c=l=G=$ $\gamma=\Omega_{k}=1$ and $q=2$ have been used here. Each of this three graphs have unphysical regions with negative Hawking temperature. The unusual things appeared in the last graph with $k=-1$. See text for the discussion about this.

When $q^{2}=2\left(-k^{2}+2 k x_{+}^{2}+3 x_{+}^{4}\right)$, or $x_{+}=\sqrt{-\frac{k}{3}+\frac{\sqrt{8 k^{2}+3 q^{2}}}{3 \sqrt{2}}}$, Hawking temperature $T=0$, and the black hole becomes an extremal one. In order for this black hole to be non-extremal we must assume

$$
q^{2}<2\left(-k^{2}+2 k x_{+}^{2}+3 x_{+}^{4}\right) .
$$

We exhibit the temperature vs. $x_{+}$curve in Figure [6, with parameters $c=l=G=\gamma=$ $\Omega_{k}=1$ and $q=2$ (Parameters are chose in the same way in all the graphical analyzes in this section, but the general properties of the physical quantities are not changed if we choose other parameters). We can see that each of the three graphs have negative values of Hawking temperature at some ranges of $x_{+}$, i.e. $q^{2}>2\left(-k^{2}+2 k x_{+}^{2}+3 x_{+}^{4}\right)$, which correspond to unphysical regions and have no physical significance. There is an infinite discontinuous point $x_{+}=x_{c}=1$ in the case of $k=-1$ (Figure 6c). When $x_{+}>x_{c}$, the temperature behaves just like the other two temperature curves in Figures 6a and 6b, gradually increasing with $x_{+}$. Another interesting property is that in the case of $k=-1$, for "small" black holes 
(with radius $0<x_{+}<1$ ), the temperature has a minimum value at the point $x_{+}=\frac{1}{\sqrt{3}}$. Remarkably, this point does not depend on the charge parameter $q$. We plot the phase structure of small black holes in Figure 7.

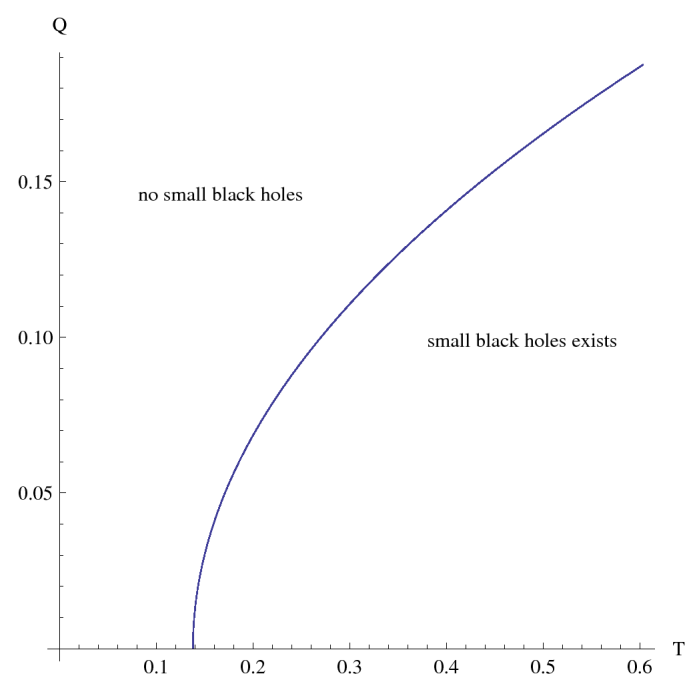

Figure 7: Phase structure of "small" $\left(0<x_{+}<1\right)$ topological black holes for $k=-1$.

As emphasized in Section III, the temperature vs. $x_{+}$curve cannot reveal all the information of the phase structure, but it tells us the "small" black holes only exists above a minimum temperature, as shown in Figure 7.

The charged topological black holes in H-L gravity have two conserved quantities $Q$ and $M$ parameterized by $q$ and $x_{+}$, just like the RN AdS black holes. The RN AdS black holes phase transitions were studied in [7, 8]. Two complementary thermodynamic ensembles were studied there, which are the grand canonical ensemble with fixed background potential and the canonical ensemble with fixed charge. In the canonical ensemble, if the fixed charge is below a critical value, with the temperature increases, small black holes are tend to become large black holes with a jump in the entropy. This is a first order phase transition with a release of "latent heat". Above the critical value of the charge, there is no phase transition. This is very similar to to the van der Waals-Maxwell liquid-gas model. This phase transition is characterized by the divergence of the specific heat with fixed charge $C_{Q}$. Recently, a new type of phase transition for the RN AdS black hole associated with the divergence of the specific heat with fixed potential $C_{\Phi}$ was suggested in [43]. The authors argued that study of the Ehrenfest's equations and the thermodynamical metrics indicate the existence of a new glassy type transition. 
Now, let's proceed to do the stability anolysis for charged topological black holes in H-L gravity. Thermodynamic stability could be studied in many different ways, depending on which thermodynamic function we choose to use. Different choices will reveal thermodynamic stability in different ensembles. A physical transparent way is to examine the sign of specific heats and other capacities. For charged topological black holes in H-L gravity in hand, four kinds of capacities can be constructed. They are

$$
\left.C_{Q} \equiv T \frac{\partial S}{\partial T}\right|_{Q},\left.\quad C_{\Phi} \equiv T \frac{\partial S}{\partial T}\right|_{\Phi},\left.\quad \tilde{C}_{T} \equiv \frac{\partial Q}{\partial \Phi}\right|_{T},\left.\quad \tilde{C}_{S} \equiv \frac{\partial Q}{\partial \Phi}\right|_{S} .
$$

The first two, i.e., the specific heats at constant electric charge or potential, determine the thermal stability of the black holes. They are positive (negative) if the black hole is thermodynamic stable (unstable) to a thermal fluctuation. The last two quantities are charge capacitances at fixed temperature or entropy. They are negative (positive) if the black hole is electrically unstable (stable) to electrical fluctuation.

Following the standard thermodynamic definition of specific heat, we calculate the specific heat of the topological charged black hole in H-L gravity for constant charge $Q$,

$$
\left.C_{Q} \equiv T \frac{\partial S}{\partial T}\right|_{Q}=\left.\frac{\partial M}{\partial T}\right|_{Q}=-\frac{\pi\left(k+x_{+}^{2}\right)^{2}\left(q^{2}+2\left(k-3 x_{+}^{2}\right)\left(k+x_{+}^{2}\right)\right) \kappa^{2} \mu^{2} \Omega_{k}}{2 \zeta_{1}\left(q^{2}+2\left(k+x_{+}^{2}\right)^{2}\right)},
$$

where

$$
\zeta_{1}=\left(k+3 x_{+}^{2}\right)
$$

Note that the expressions of $C_{Q}$ and $T$ share the same factor $q^{2}+2\left(k-3 x_{+}^{2}\right)\left(k+x_{+}^{2}\right)$ in their numerator, and the non-extremal condition will make $C_{Q}$ positive definite in the physical parameters region when $k=0,1$, which means they are thermodynamic stable locally.

The specific heat for constant potential $\Phi$ is given by

$$
\left.C_{\Phi} \equiv T \frac{\partial S}{\partial T}\right|_{\Phi}=-\frac{\pi\left(k+x_{+}^{2}\right)^{2}\left(-q^{2}+2\left(k-3 x_{+}^{2}\right)\left(k+x_{+}^{2}\right)\right) \kappa^{2} \mu^{2} \Omega_{k}}{2 \zeta_{2}},
$$

where

$$
\zeta_{2}=\left(2 k^{3}-k q^{2}+10 k^{2} x_{+}^{2}+q^{2} x_{+}^{2}+14 k x_{+}^{4}+6 x_{+}^{6}\right)
$$

We can also calculate the charge capacitances at fixed temperature or entropy

$$
\left.\tilde{C}_{T} \equiv \frac{\partial Q}{\partial \Phi}\right|_{T}=\frac{1}{16 l^{2}}\left(x_{+}+\frac{2 q^{2} x_{+}\left(k+x_{+}^{2}\right)}{\zeta_{2}}\right) \kappa^{2} \mu^{2} \Omega_{k}
$$




$$
\left.\tilde{C}_{S} \equiv \frac{\partial Q}{\partial \Phi}\right|_{S}=\frac{1}{16 l^{2}} x_{+} \kappa^{2} \mu^{2} \Omega_{k}
$$

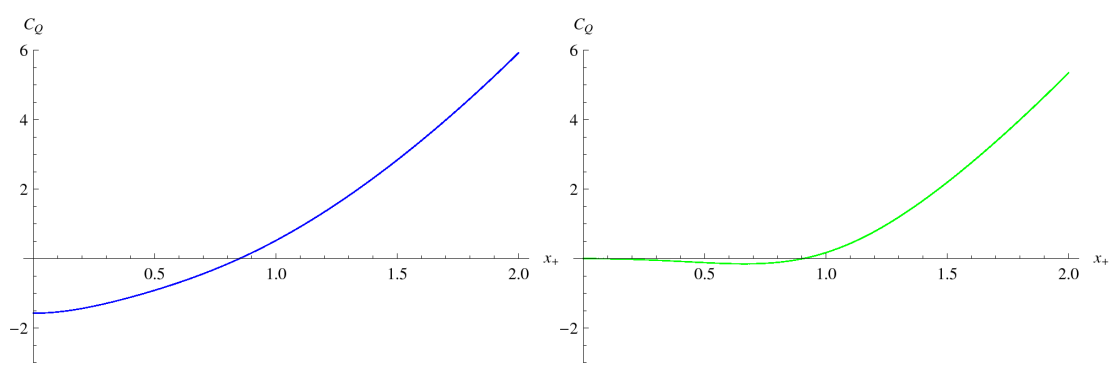

(a) $k=1$

(b) $k=0$

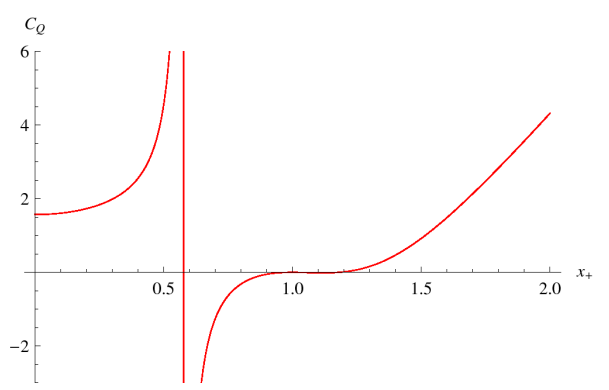

(c) $k=-1$

Figure 8: Specific heat for constant charge $Q$ vs. $x_{+}$, with $c=l=G=\gamma=\Omega_{k}=1$ and $q=2 . C_{Q}$ is positive definite in the physical region of parameters when $k=0,1$, it can change it's sign at a parameter $q$ independent threshold point $x_{+}=\frac{1}{\sqrt{3}}$ in the case of $k=-1$.

We plot $C_{Q}$ in Figure 8, with parameters $c=l=G=\gamma=\Omega_{k}=1$ and $q=2$. As we can see from Figure $8 \mathrm{c}$, the specific heat passes from positive to negative values through an infinite discontinuity, and then passes across the horizontal ordinate again continuously (a small range of $x_{+}$bigger than $x_{+}=1$ is unphysical since the Hawking temperature is negative). The first threshold point is associated with a local phase transition, and the latter happens at Hawking temperature $T=0$ where the black hole becomes an extremal one. The latter threshold point also occur in the cases of $k=1$ and $k=0$. These points are not associated with phase transitions. However, they define the unphysical range of $x_{+}$, where the temperature acquires a negative value and the curve does not have any physical significance. The strange behavior of the specific heat for $k=-1$ should be emphasized. 
$C_{Q}$ has a divergent point in the case of $k=-1$ at $\zeta_{1}=0$, i.e., $x_{+}=\frac{1}{\sqrt{3}}$, which is consistent with the minimum of temperature. This indicates a local phase transition.

For brevity, we are not going to show all the other three $C$ 's in graphs. We can see that $C_{Q}, C_{\Phi}$ and $\tilde{C}_{T}$ all can have both positive and negative values in certain parameter regions, they will change signs in threshold points. The threshold points are at certain value of $x_{+}$, $p$ and $k$ which satisfy the condition

$$
\zeta_{1}=0 \quad \text { or } \quad \zeta_{2}=0
$$

These threshold points signal the change of local stabilities and hence are typically associated with certain local phase transitions. $\tilde{C}_{S}$ is positive definite, so it may not be relevant to phase transition.

The free energy can reveal global stability properties of black holes. It is straightforward to calculated as

$$
\begin{aligned}
F & =M-T S \\
& =\frac{\left.\kappa^{2} \mu^{2} \Omega_{k}\left(4 k^{3}+14 k^{2} x_{+}^{2}+3 q^{2} x_{+}^{2}-2 x_{+}^{6}+2 k \ln \left(x_{+}\right)\left(2 k^{2}+q^{2}-4 k x_{+}^{2}-6 x_{+}^{4}\right)+2 k\left(q^{2}+4 x_{+}^{4}\right)\right)_{25}\right)}{64 l^{2} x_{+}\left(k+x_{+}^{2}\right)}
\end{aligned}
$$

We plot the free energy as a function of $x_{+}$in Figure 9, with $c=l=G=\gamma=\Omega_{k}=1$ and $q=2$. For $k=0,1$, the free energy decrease steadily in the physical region. Any non-extremal black holes have positive heat capacity, and the black holes are stable. Now, let's focus on Figure 9c, For "small" black holes in the case of $k=-1$, the free energy has a maximum at $x_{+}=\frac{1}{\sqrt{3}}$, which is also the point of minimum temperature and divergent heat capacity $C_{Q}$. As we can see from Figure $8 \mathrm{c}$, there are three branches of black holes. In the region $\frac{1}{\sqrt{3}}<x_{+}<1$, the middle branch non-extremal black holes have negative value of $C_{Q}$, and they are local unstable, while the small and large branches are local stable. One may suspect that there is a global phase transition between the small and large $x_{+}$black holes. This does not take place since the large branch of black holes persist to dominate the thermodynamics at all temperatures. As we can see from Figure 10, at all temperatures, the large branch (solid curve in the graph) has the most negative value of free energy, and global phase transition can not comes up. 


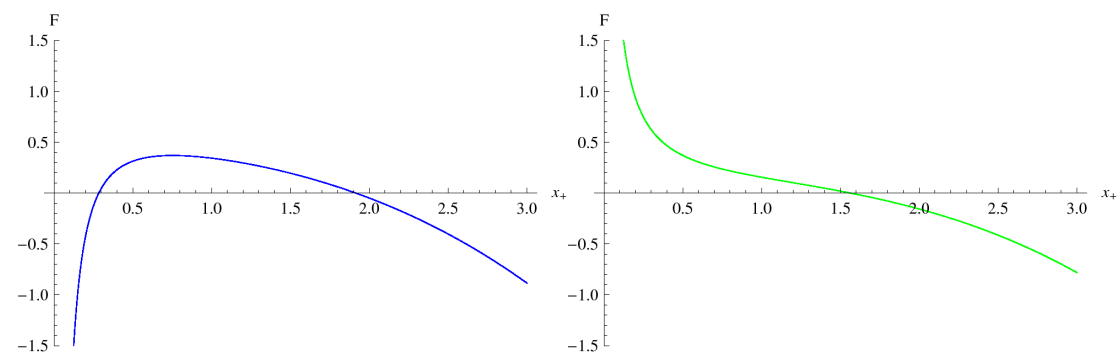

(a) $k=1$

(b) $k=0$

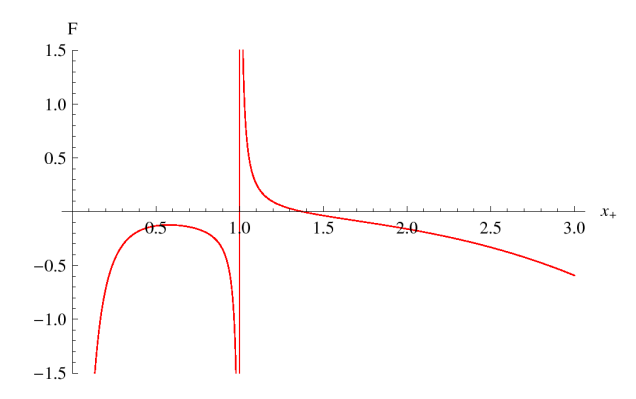

(c) $k=-1$

Figure 9: Free energy for constant charge $Q$ vs. $x_{+}$, the values $c=l=G=\gamma=\Omega_{k}=1$ and $q=2$ have been used here, see text for discussion.

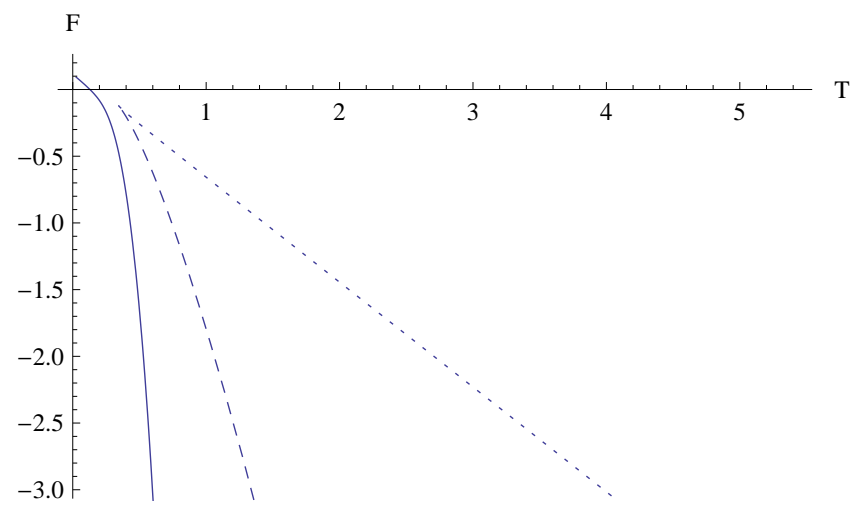

Figure 10: Free energy for charged topological black holes with $k=-1$ vs. temperature, with $c=l=G=\gamma=\Omega_{k}=1$ and $q=2$. The dotted curve is for the middle branch, while the dashed and solid curves are associated with the small and large branches, respectively.

An alternative way to study thermodynamics is using thermodynamical metrics, which have been introduced in Section [1. Next, we are going to study the properties of the corresponding state space geometry. The Ruppeiner and Weinhold metrics, and the thermody- 
namical metric derived from the free energy $F=M-T S$ are defined by

$$
\begin{array}{ll}
\vec{M}=\left\{M^{i}\right\} \equiv\left\{M, N^{a}\right\} \quad, & g_{i j}^{(S)} \equiv \frac{\partial^{2} S\left(M, N^{a}\right)}{\partial M^{i} \partial M^{j}}, \\
\vec{S}=\left\{S^{i}\right\} \equiv\left\{S, N^{a}\right\} & g_{i j}^{(M)} \equiv \frac{\partial^{2} M\left(S, N^{a}\right)}{\partial S^{i} \partial S^{j}}, \\
\vec{T}=\left\{T^{i}\right\} \equiv\left\{T, N^{a}\right\} \quad, \quad g_{i j}^{(F)} \equiv \frac{\partial^{2} F\left(T, N^{a}\right)}{\partial T^{i} \partial T^{j}} .
\end{array}
$$

The Ricci scalars of the above thermodynamical metrics can be calculated parallel to [31]. The thermodynamical curvatures are too complicated to present, so we only give the denominators of the Ricci scalars here, which are enough for us to analyze their singular behaviors. The denominators of the Ricci scalars, denoted by $D(R)$, are given by

$$
\begin{aligned}
D\left(R^{(S)}\right)= & \zeta_{2} \pi \kappa^{2} \mu^{2} \Omega_{k}\left(k+x^{2}\right)\left(8+q^{2}-8 x_{+}^{2}-6 x_{+}^{4}\right)^{4}\left(2+q^{2}-4 x_{+}^{2}-6 x_{+}^{4}\right)^{4}\left(2+3 x_{+}^{2}+x_{+}^{4}\right)^{2} \\
& \left(q^{2}\left(-1+x_{+}^{2}\right)+2\left(1+x_{+}^{2}\right)^{2}\left(1+3 x_{+}^{2}\right)\right)^{2}\left(q^{2}\left(-2+x_{+}^{2}\right)+2\left(2+x_{+}^{2}\right)^{2}\left(2+3 x_{+}^{2}\right)\right)^{2}(29 \\
D\left(R^{(M)}\right)= & \frac{\kappa^{2} \mu^{2} \Omega_{k}}{l^{2}} \zeta_{2}\left(q^{2}\left(-1+x_{+}^{2}\right)+2\left(1+x_{+}^{2}\right)^{2}\left(1+3 x_{+}^{2}\right)\right)^{2} \\
& \left(q^{2}\left(-2+x_{+}^{2}\right)+2\left(2+x_{+}^{2}\right)^{2}\left(2+3 x_{+}^{2}\right)\right)^{2} \\
D\left(R^{(F)}\right)= & \frac{\kappa^{2} \mu^{2} \Omega_{k}}{l^{2}}\left(q^{2}+2\left(1+x_{+}^{2}\right)^{2}\right)^{4}\left(q^{2}+2\left(2+x_{+}^{2}\right)^{2}\right)^{4}
\end{aligned}
$$

We can see that the curvature singularity of these metrics are related to the local phase transition associated with the vanishing of $\zeta_{2}$, not $\zeta_{1}$. In the cases discussed in [31], the curvature singularity of the Ricci scalar for the free-energy metric $R^{(F)}$ is associated with the divergence of the specific heat at $\zeta_{1}=0$. However, this does not happen in the case of topological charged black hole in H-L gravity. The denominator of $C_{Q}$ and $R^{(F)}$ indeed share the same factor

$$
\zeta_{3}=q^{2}+2\left(k+x_{+}^{2}\right)^{2}
$$

But $\zeta_{3}$ does not vanish unless we set $q=0$, which reduce to the uncharged case discussed in Sec.(III). So we have to declare that one exception to the framework in [31] has been found. The specific heat $C_{Q}$ of charged black hole in H-L gravity with $k=-1$ is divergent at $x_{+}=\frac{1}{\sqrt{3}}$, but none of the three Ricci scalars have singularity at this point. The violation of the correspondence only exists when $k=-1$, this happens because the topology of its event horizon is non-spherical. This is a very interesting feature of H-L gravity. 
In closing this section, we would like to stress the unusual features we have found here. The phase structures is vivid in the charged topological black holes in H-L gravity, which are very different from the RN AdS black holes in Einstein gravity. For the charged black holes with sphere topology in H-L gravity, there is no phase transition in the canonical ensemble with fixed charge, since the specific heat with fixed charge $C_{Q}$ is positive definite, and all the non-extremal black holes can local stable exist at all temperatures. Whilst, this is not the performance of RN AdS black holes. Another difference is that the thermodynamical metrics can not reveal all of the local phase transition signals in H-L gravity, such as the charged black holes in H-L gravity with non-spherical event horizon. One should also note that this is a local phase transition, global phase transitions are not take place in the canonical ensemble with fixed charge for the charged topological black holes in H-L gravity. As we have declared in the end of Section III, the ultraviolet behavior of spacetime in H-L gravity deserves as the reason for all of those strange properties in black hole thermodynamics.

\section{KIHAGIAS-SFETOSOS BLACK HOLE}

The action of the IR modified Hořava-Lifshitz gravity is obtained by adding a term $\mu^{4} R^{(3)}$ to the original Hořava-Lifshitz action[37], in order to get a Minkowski vacuum in IR. The Kihagias-Sfetosos (KS) black hole is a solution of the IR modified (or deformed) HořavaLifshitz gravity, which was originally obtained in [37]. The thermodynamical properties of this kind of black holes has been studied in [41, 44-46]. In this section, we shall study phase transitions in KS black holes. Stability analysis and state space geometry will both be used. The solution of KS black hole is defined by the line element

$$
d s^{2}=-N^{2}(r) d t^{2}+\frac{1}{f(r)} d r^{2}+r^{2}\left(d \theta^{2}+\sin ^{2} \theta d \phi^{2}\right)
$$

where

$$
N^{2}=f=1+\omega r^{2}-\sqrt{r\left(\omega^{2} r^{3}+4 \omega m\right)}
$$

and $\omega=\frac{16 \mu^{2}}{\kappa^{2}}$ is a combined parameter of the original parameters $\kappa$ and $\mu$ in $\mathrm{H}-\mathrm{L}$ gravity

action. The quantity $\sqrt{\frac{1}{2 \omega}}$ behaves as a charge-like parameter, we denote $P=\sqrt{\frac{1}{2 \omega}}$ and consider it as a new parameter in the black hole thermodynamics. Now, we can rewrite (34) as

$$
N^{2}(r, P)=f(r, P)=1+\frac{r^{2}}{2 P^{2}}-\sqrt{\frac{r^{4}}{4 P^{4}}+\frac{2 m r}{P^{2}}}
$$


The horizons of KS black hole is determined by $f(r, P)=0$, which gives

$$
r_{ \pm}=m \pm \sqrt{m^{2}-P^{2}}
$$

The KS black hole is asymptotically flat, and it's thermodynamic properties will change dramatically from the charged topological black holes in H-L gravity. The mass parameter $m$ and temperature of the KS black hole can be expressed respectively as

$$
\begin{aligned}
& m=\frac{r_{+}}{2}+\frac{P^{2}}{2 r_{+}}=\frac{r_{+}+r_{-}}{2}, \\
& T=\left.\frac{f^{\prime}(r)}{4 \pi}\right|_{r=r_{+}}=\frac{r_{+}^{2}-P^{2}}{4 \pi r_{+}\left(2 P^{2}+r_{+}^{2}\right)}=\frac{r_{+}-r_{-}}{4 \pi r_{+}\left(r_{+}+2 r_{-}\right)} .
\end{aligned}
$$

The KS black hole becomes an extreme one when $r_{+}=r_{-}$, i.e., $m=r_{+}=|P|$. If one regard $m$ as the mass of KS black hole, using the first law $d m=T d S$, the entropy can be obtained via integration

$$
S=\pi r_{+}^{2}+2 \pi P^{2} \ln r_{+}^{2}+S_{0}
$$

This is an entropy with logarithmic term. There are many discussions for Eq.(39). In [41], the authors regard the existence of logarithmic term in the entropy as a unique feature of the Hořava-Lifshitz gravity. In [45], it was suggested that this logarithmic term can be interpreted as the GUP-inspired black hole entropy, and a duality between the entropy of the KS black hole and the GUP-inspired Schwarzschild black hole has been shown. On the other hand, one may treat $m$ as a mass parameter and use the ADM mass to write its first law of thermodynamics. The authors of [47] and [48] argued that one should use the area-law entropy and ADM mass, which also satisfy the first law of thermodynamics. Here, we examine the thermodynamical properties of KS black holes by using the first idea, and the latter idea will be adopt in the appendix for completeness.

If we take $P$ as the $\mathrm{KS}$ black hole charge, the potential $\Phi$ corresponds to $P$ is

$$
\Phi=\frac{P\left(2 P^{2}+r_{+}^{2}+\left(P-r_{+}\right)\left(P+r_{+}\right) \ln \left[r_{+}^{2}\right]\right)}{2 P^{2} r_{+}+r_{+}^{3}}
$$

Those thermodynamical quantities also satisfy the first law of thermodynamic $d m=T d S+$ $\Phi d P$. 


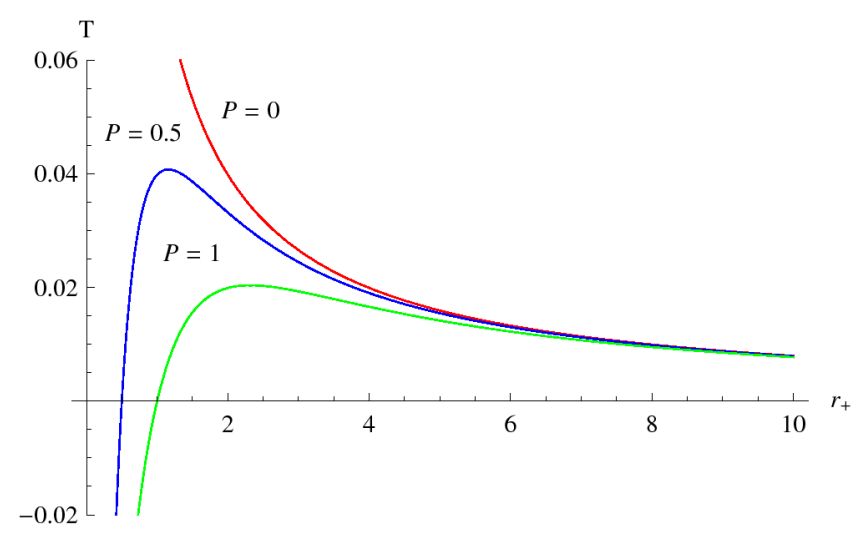

Figure 11: Temperature of KS black holes.

The Hawking temperature of KS black hole behaves very similar to RN black hole. As depicted in Figure 11, the KS black hole has a maximum temperature for $P \neq 0$, when $2 P^{4}+5 P^{2} r_{+}^{2}-r_{+}^{4}=0$, i.e.,

$$
r_{+}=r_{m}=\sqrt{\frac{(5+\sqrt{33}) P^{2}}{2}}
$$

which satisfies $d T / d r_{+}=0$. And we will found that it is just the threshold point for specific heat changing from negative to positive values through an infinite discontinuity. Hence, for $P \neq 0$, the point at $r_{m}$ may signal a local phase transition. We plot the phase structure for KS black holes in Figure 12. We can find that there is another similarity between RN black holes and KS black holes that both of these black holes can exist at all temperatures.

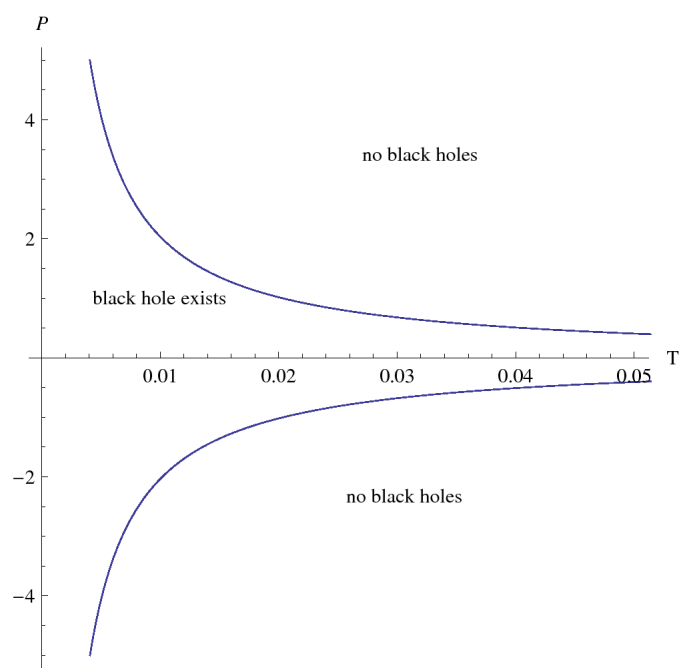

Figure 12: Phase structure of KS black holes. KS black holes can exist at all temperatures.

Taking $P$ and $r_{+}$as variables, we can calculate the specific heat for either constant $P$ or 
constant potential $\Phi$, and charge capacitances defined in (18). They are given by

$$
\begin{aligned}
C_{P} & \left.\equiv T \frac{\partial S}{\partial T}\right|_{P}=\left.\frac{\partial m}{\partial T}\right|_{P}=\frac{2 \pi\left(-4 P^{6}+3 P^{2} r_{+}^{4}+r_{+}^{6}\right)}{\xi_{1}} \\
C_{\Phi} & \left.\equiv T \frac{\partial S}{\partial T}\right|_{\Phi} \\
& =\left.\frac{\left(2 \pi\left(2 P^{2}+r_{+}^{2}\right)\left(-4 P^{6}+12 P^{4} r_{+}^{2}+9 P^{2} r_{+}^{4}+r_{+}^{6}+\left(2 P^{6}+7 P^{4} r_{+}^{2}+4 P^{2} r_{+}^{4}-r_{+}^{6}\right) \ln \left(r_{+}^{2}\right)\right)\right)_{(43)}\left(P^{2}-r_{+}^{2}\right) \xi_{2}}{\tilde{C}_{T}} \equiv \frac{\partial Q}{\partial \Phi}\right|_{T}=\frac{4 P^{6} r_{+}+12 P^{4} r_{+}^{3}+3 P^{2} r_{+}^{5}-r_{+}^{7}}{\left(P^{2}-r_{+}^{2}\right) \xi_{2}} \\
\tilde{C}_{S} & \left.\equiv \frac{\partial Q}{\partial \Phi}\right|_{S}=\frac{\left(r_{+}\left(2 P^{2}+r_{+}^{2}\right)^{3}\right)}{\xi_{3}}
\end{aligned}
$$

where

$$
\begin{aligned}
& \xi_{1}=2 P^{4}+5 P^{2} r_{+}^{2}-r_{+}^{4} \\
& \xi_{2}=4 P^{4}+16 P^{2} r_{+}^{2}+r_{+}^{4}+\left(2 P^{4}+5 P^{2} r_{+}^{2}-r_{+}^{4}\right) \ln \left(r_{+}^{2}\right) \\
& \xi_{3}=\left(2 P^{2}+r_{+}^{2}\right)^{3}+\ln \left(r_{+}^{2}\right)\left(4 P^{6}+24 P^{4} r_{+}^{2}+9 P^{2} r_{+}^{4}-r_{+}^{6}+2\left(2 P^{6}+5 P^{4} r_{+}^{2}-P^{2} r_{+}^{4}\right) \ln \left(r_{+}^{2}\right)\right)(
\end{aligned}
$$

Since $r_{+}^{2}>P^{2}$ for a non-extremal KS black hole, the numerator of $C_{P}$ is positive definite, and it is divergent only when $\xi_{1}=0$. This happens at $x_{+}=x_{m}$ which is just the maximum point of temperature, as we showed in Eq.(41) and Figure 11, A graph of specific heat $C_{P}$ is shown in Figure 13 .

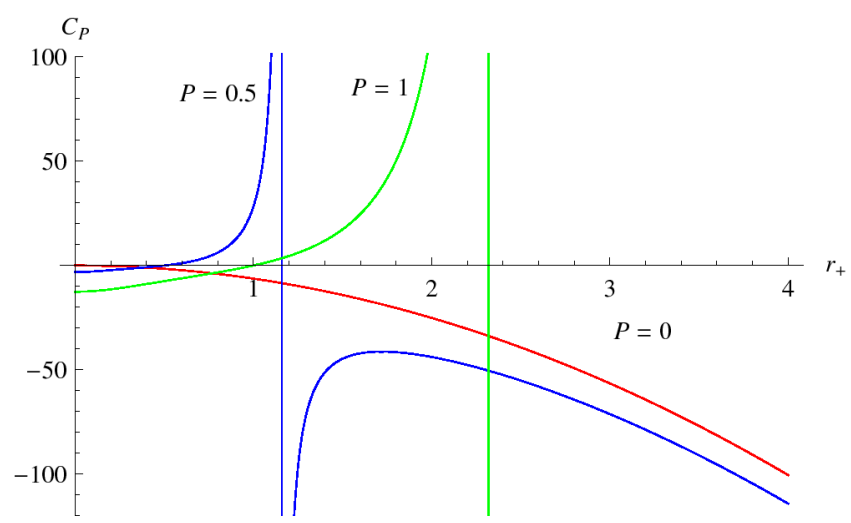

Figure 13: Heat capacity of KS black holes.

One can see from Figure 13 that there always exist threshold points when $P \neq 0$. A more recent paper argued that this divergent points of $C_{P}$ is associated to a second-order phase transition[49]. 
We also plot the free energy

$$
F=m-T S=\frac{4 P^{4}+7 P^{2} r_{+}^{2}+r_{+}^{4}+2 \ln \left(r_{+}^{2}\right)\left(P^{4}-P^{2} r_{+}^{2}\right)}{4\left(2 P^{2} r_{+}+r_{+}^{3}\right)},
$$

in Figure 14. We see that the free energy for $P \neq 0$ has a local maximum at $\xi_{1}=0$.

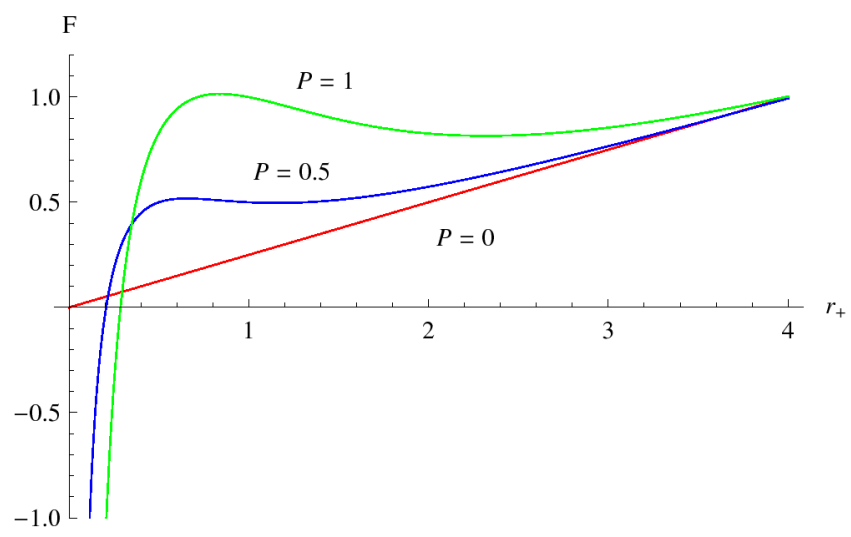

Figure 14: Free energy of KS black holes.

The above results can be interpreted as follow. The specific heat with constant $P$ is positive for small radius KS black holes, while negative for a larger one. This indicates that the small KS black holes are thermodynamic stable, while the large KS black holes are unstable. It is nothing out of ordinary for a asymptotically flat black hole to have negative specific heat since the Schwarzschild black hole in Einstein gravity is asymptotically flat spacetime, it has negative specific heat and is unstable. However it is unusual that the small KS black holes are stable. This conclusion is also supported by Figure 14, since the unstable large KS black holes have positive free energy while the stable small KS black holes can have negative free energy when the radius is small enough. $P=\sqrt{\frac{\kappa^{2}}{32 \mu^{2}}} \neq 0$ is the crucial condition for this interesting phase structure in KS black holes, which means the high derivative term in the $\mathrm{H}-\mathrm{L}$ gravity action comes into play, and change the thermodynamic properties of asymptotically flat black holes.

Other three $C$ 's all can have both positive and negative values in suitable parameter regions. The threshold points are at certain value of $P$ and $r_{+}$which satisfy the condition

$$
\xi_{2}=0 \quad \text { or } \quad \xi_{3}=0
$$

The behaviors of $C_{\Phi}, \tilde{C}_{T}$, and $\tilde{C}_{S}$ will reveal thermodynamic stability in different ensembles, interesting phase structures in the KS black holes can be found by examine this quantities. 
Thermodynamical metrics can be constructed as we did in Section [II, and it is straightforward to calculate Ricci scalars of thermodynamical metrics. Considering the complexity of the expression of those Ricci scalars, we only show the denominators. They are given by

$$
\begin{aligned}
D\left(R^{(S)}\right) & =\pi \xi_{2}^{2} \\
D\left(R^{(M)}\right) & =\left(P-r_{+}\right)^{2}\left(P+r_{+}\right)^{2}\left(2 P^{2}+r_{+}^{2}\right) \xi_{2}^{2} \\
D\left(R^{(F)}\right) & =\xi_{1}^{2} \xi_{3}^{2}
\end{aligned}
$$

We see that all possible phase transitions correspond to curvature singularities of certain thermodynamical metrics, which are consistent to the framework proposed by [31].

\section{CONCLUSION}

Black hole phase transitions have been extensively studied since the discovery of HawkingPage phase transition and Witten's interpretation of the transition in the frame of AdS/CFT correspondence. However, the lack of exact knowledge about the microscopic statistical framework underlying black hole thermodynamic renders the issue of phase transitions in black holes far from being completely settled. In spite of these difficulties, some local and global stability properties were examined in the constructed canonical ensemble or microcanonical ensemble. Local stability in the canonical ensemble is equivalent to the positivity of the heat capacity. However, the local stability is not sufficient to ensure global stability, there do exist regions which are locally stable but globally unstable, as we found in this paper.

In this paper, we have studied black hole phase transitions in (deformed) Hořava-Lifshitz gravity though stability analyses, including the uncharged/charged topological black hole and KS black hole. Some interesting observations have been made. Compared to the Einstein gravity, the phase structure of black holes in H-L gravity changed dramatically, the stability of small radius black holes are essential different. Pursuing it's deep reason of this will help us to gain some information about how gravity works in small scale spacetime.

The framework proposed in [31] is also associated with the local stability analyses to black hole phase transitions. It is still unclear whether this framework can uncover some global stability properties of black holes. We found a probable counter-example to this 
framework. There is an infinite discontinuity at the specific heat curve for charged black hole with hyperbolic event horizon in H-L gravity. However, this discontinuity does not have a corresponding curvature singularities of thermodynamical metrics. The violation of the correspondence in H-L gravity may be due to the non-spherical topology of their event horizons. We guess that the topology of the event horizon and the dimension of spacetime may influence the validity of the correspondence. One should also note that this is associated with a local phase transition, not a global phase transition.

\section{Acknowledgments}

We thank Y.J.Du, J.L.Li, Q.Ma and Y.Q.Wang for useful discussions. Chen would like to thank the organizer and the participants of the advanced workshop, "Dark Energy and Fundamental Theory" supported by the Special Fund for Theoretical Physics from the National Natural Science Foundation of China with grant No. 10947203, for stimulating discussions and comments. The research is supported by the NNSF of China Grant No. 11075138, No. 10775116, 973 Program Grant No. 2005CB724508.

\section{Appendix}

For KS black hole, there is another idea in defining the mass and entropy. One can treat $m$ as a mass parameter, and to use the one quarter area entropy and ADM mass[47, 48], In this appendix, we will adopt this idea to investigate the thermodynamic properties of KS black holes. The expressions of entropy and ADM mass are given by

$$
\begin{gathered}
S=\frac{A}{4}=\pi r_{+}^{2}, \\
M=\frac{r_{+}}{2}-\frac{3 \tan ^{-1}\left(\sqrt{\omega} r_{+}\right)}{4 \sqrt{\omega}}+\frac{3 \pi}{8 \sqrt{\omega}} .
\end{gathered}
$$

The mass parameter $m$ is Eq.(37), and temperature formula is the same as Eq.(38), discussions about this can be found in Section IV.

The potential $V$ corresponding to the charge like parameter $P$ is computed as

$$
V=-\frac{3\left(\left(1+\omega r_{+}^{2}\right) \tan ^{-1}\left(\sqrt{\omega} r_{+}\right)-\sqrt{\omega} r_{+}\right.}{2 \sqrt{2}\left(1+\omega r_{+}^{2}\right)}+\frac{3 \sqrt{2} \pi}{8}
$$


which satisfy the Smarr law

$$
M=2 T S+V P
$$

and first law of thermodynamics

$$
d M=T d S+V d P
$$

Furthermore, one can calculate the specific heats for constant $P$ or constant potential $V$,

$$
\begin{gathered}
C_{P}=\left.T \frac{\partial S}{\partial T}\right|_{P}=\left.\frac{\partial M}{\partial T}\right|_{P}=\frac{2 \pi r_{+}^{2}\left(-2 P^{4}+P^{2} r_{+}^{2}+r_{+}^{4}\right)}{2 P^{4}+5 P^{2} r_{+}^{2}-r_{+}^{4}}, \\
C_{V}=\left.\frac{\partial M}{\partial T}\right|_{V}=\frac{\pi r_{+}\left(3 \sqrt{2} \pi-6 \sqrt{2} \tan ^{-1}\left(\frac{\sqrt{\frac{1}{P^{2}}} r_{+}}{\sqrt{2}}\right)+4 \sqrt{\frac{1}{P^{2}}} r_{+}\right)\left(2 P^{2}+r_{+}^{2}\right)}{2 \sqrt{\frac{1}{P^{2}}}\left(P^{2}-r_{+}^{2}\right)} .
\end{gathered}
$$

The capacitances at a fixed temperature or entropy are given respectively by

$$
\begin{gathered}
\tilde{C}_{T}=\left.\frac{\partial P}{\partial V}\right|_{T}=\frac{\sqrt{\frac{1}{P^{2}}} P\left(2 P^{2}+r_{+}^{2}\right)\left(2 P^{4}+5 P^{2} r_{+}^{2}-r_{+}^{4}\right)}{3 r_{+}^{3}\left(P^{2}-r_{+}^{2}\right)}, \\
\tilde{C}_{S}=\left.\frac{\partial P}{\partial V}\right|_{S}=\frac{\sqrt{\frac{1}{P^{2}}} P\left(2 P^{2}+r_{+}^{2}\right)^{2}}{3 r_{+}^{3}} .
\end{gathered}
$$

The feature of the heat capacity can be seen from Figure 15. $C_{P}$ also has a pole when $\xi_{1}=0$, which agrees with what we get from Eq.(42). Also note that the divergence of $C_{P}$ is associated with the vanishing of $\tilde{C}_{T}$. But the other three $C$ 's are very different from what we discussed in Section IV] $C_{V}$ and $\tilde{C}_{T}$ both have singularity at $P^{2}=r_{+}^{2}$, or $P^{2}=m^{2}$ where the black hole is extremal, which may be the critical point for a phase transition from extremal to non-extremal black hole. $\tilde{C}_{S}$ is not divergent in the physical region of parameters, and is not relevant to KS black hole phase transition.

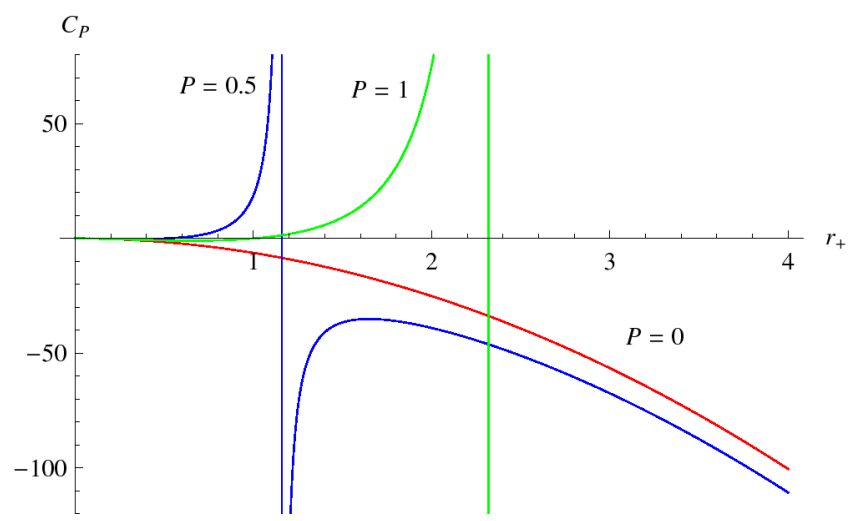

Figure 15: Heat capacity for KS black hole with area entropy. 
The free energy

$$
\begin{aligned}
F & =M-T S \\
& =\frac{1}{8}\left(\frac{3 \sqrt{2} \pi-6 \sqrt{2} \tan ^{-1}\left(\frac{\sqrt{\frac{1}{P^{2}}} r_{+}}{\sqrt{2}}\right)+4 \sqrt{\frac{1}{P^{2}}} r_{+}}{\sqrt{\frac{1}{P^{2}}}}+\frac{2 r_{+}\left(P^{2}-r_{+}^{2}\right)}{2 P^{2}+r_{+}^{2}}\right) \\
& =\frac{1}{8}\left(3 \sqrt{2}|P|\left(\pi-2 \tan ^{-1}\left(\frac{r_{+}}{\sqrt{2}|P|}\right)\right)+4 r_{+}+\frac{2 r_{+}\left(P^{2}-r_{+}^{2}\right)}{2 P^{2}+r_{+}^{2}}\right)
\end{aligned}
$$

is plotted in Figure 16.

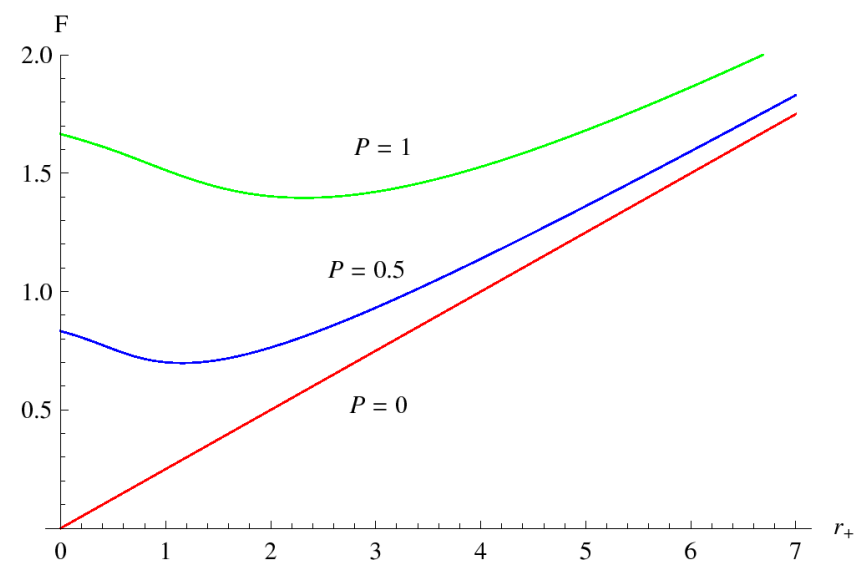

Figure 16: Free energy for KS black hole with are entropy.

The minimum of free energy corresponds to the divergent point of $C_{P}$, and the maximum point of temperature.

The Ricci scalars of the the Ruppeiner and Weinhold metrics, and the thermodynamical metric derived from the free energy are given by

$$
\begin{gathered}
R^{(S)}=0 \\
R^{(M)}=-\frac{2\left(2 P^{4}+r_{+}^{4}\right)}{r_{+}\left(P^{2}-r_{+}^{2}\right)^{2}}, \\
R^{(F)}=-\frac{4\left(2 P^{2}+r_{+}^{2}\right)^{2}\left(2 P^{4}+r_{+}^{4}\right)}{r_{+}\left(-2 P^{4}-5 P^{2} r_{+}^{2}+r_{+}^{4}\right)^{2}} .
\end{gathered}
$$

It is not surprise that the Ruppeiner curvature $R^{(S)}$ vanishes, since we have treating the KS black hole similar with the RN black hole, while the Ruppeiner curvature for the latter is zero. 
We see that the divergent point of $R^{(M)}$ corresponds to the divergent point of $C_{V}$ and $\tilde{C}_{T}$, and that the divergent point of $R^{(F)}$ correspond to the divergent point of $C_{P}$. The results also agree with the analyzes in [31].

[1] Jacob D. Bekenstein. Black holes and entropy. Phys. Rev., D7:2333-2346, 1973.

[2] S. W. Hawking. Particle Creation by Black Holes. Commun. Math. Phys., 43:199-220, 1975.

[3] JM Bardeen, B. Carter, and SW Hawking. The four laws of black hole mechanics. Communications in Mathematical Physics, 31(2):161-170, 1973.

[4] SW Hawking and D.N. Page. Thermodynamics of black holes in anti-de Sitter space. Communications in Mathematical Physics, 87(4):577-588, 1983.

[5] E. Witten. Anti-de Sitter space, thermal phase transition, and confinement in gauge theories. Adv. Theor. Math. Phys., 2(3):505-532, 1998.

[6] PCW Davies. Thermodynamic phase transitions of Kerr-Newman black holes in de Sitter space. Classical and Quantum Gravity, 6:1909, 1989.

[7] A. Chamblin, R. Emparan, C.V. Johnson, and R.C. Myers. Charged AdS black holes and catastrophic holography. Physical Review D, 60(6):64018, 1999.

[8] A. Chamblin, R. Emparan, C.V. Johnson, and R.C. Myers. Holography, thermodynamics, and fluctuations of charged AdS black holes. Physical Review D, 60(10):104026, 1999.

[9] M.M. Caldarelli, G. Cognola, and D. Klemm. Thermodynamics of Kerr-Newman-AdS black holes and conformal field theories. Classical and Quantum Gravity, 17:399, 2000.

[10] B.M.N. Carter and I.P. Neupane. Thermodynamics and stability of higher dimensional rotating (Kerr-) AdS black holes. Physical Review D, 72(4):43534, 2005.

[11] R.G. Cai. Gauss-Bonnet black holes in AdS spaces. Physical Review D, 65(8):84014, 2002.

[12] R.G. Cai, S.P. Kim, and B. Wang. Ricci flat black holes and Hawking-Page phase transition in Gauss-Bonnet gravity and dilaton gravity. Physical Review D, 76(2):24011, 2007.

[13] Y.S. Myung. Phase transition for black holes with scalar hair and topological black holes. Physics Letters B, 663(1-2):111-117, 2008.

[14] T.K. Dey, S. Mukherji, S. Mukhopadhyay, and S. Sarkar. Phase transitions in higher derivative gravity. Journal of High Energy Physics, 04:014, 2007.

[15] A. Sheykhi. THERMODYNAMICAL PROPERTIES OF TOPOLOGICAL BORN- INFELD- 
DILATON BLACK HOLES. International Journal of Modern Physics D, 18(1):25-42, 2009.

[16] S. Fernando. Thermodynamics of Born-Infeld-anti-de Sitter black holes in the grand canonical ensemble. Physical Review D, 74(10):104032, 2006.

[17] Y.S. Myung, Y.W. Kim, and Y.J. Park. Thermodynamics and phase transitions in the BornInfeld-anti-de Sitter black holes. Physical Review D, 78(8):84002, 2008.

[18] D. Anninos and G. Pastras. Thermodynamics of the Maxwell-Gauss-Bonnet anti-de Sitter black hole with higher derivative gauge corrections. Journal of High Energy Physics, 07:030, 2009 .

[19] MH Dehghani and R. Pourhasan. Thermodynamic instability of black holes of third order Lovelock gravity. Physical Review D, 79(6):64015, 2009.

[20] PCW Davies. The thermodynamic theory of black holes. Proceedings of the Royal Society of London. Series A, Mathematical and Physical Sciences, 353(1675):499-521, 1977.

[21] PCW Davies. Thermodynamics of black holes. Reports on Progress in Physics, 41:1313, 1978.

[22] D. Birmingham. Topological black holes in anti-de Sitter space. Classical and Quantum Gravity, 16:1197, 1999.

[23] G. Koutsoumbas, E. Papantonopoulos, and G. Siopsis. Phase transitions in charged topological-AdS black holes. Journal of High Energy Physics, 05:107, 2008.

[24] Weinhold.F. J.Chem.Phys, 63:2479, 1975.

[25] G. Ruppeiner. Thermodynamics: A Riemannian geometric model. Physical Review A, 20(4):1608-1613, 1979.

[26] G. Ruppeiner. Riemannian geometry in thermodynamic fluctuation theory. Reviews of Modern Physics, 67(3):605-659, 1995.

[27] J.E. Åman, I. Bengtsson, and N. Pidokrajt. Geometry of black hole thermodynamics. General Relativity and Gravitation, 35(10):1733-1743, 2003.

[28] J. Shen, R.G. Cai, B. Wang, and R.K. Su. Thermodynamic geometry and critical behavior of black holes. International Journal of Modern Physics A, 22(1):11-28, 2007.

[29] G. Ruppeiner. Thermodynamic curvature and phase transitions in Kerr-Newman black holes. Physical Review D, 78(2):24016, 2008.

[30] A. Sahay, T. Sarkar, and G. Sengupta. Thermodynamic geometry and phase transitions in Kerr-Newman-AdS black holes. Journal of High Energy Physics, 04(4):118, 2010.

[31] Haishan Liu, H. Lu, Mingxing Luo, and Kai-Nan Shao. Thermodynamical Metrics and Black 
Hole Phase Transitions. JHEP, 12:054, 2010, 1008.4482.

[32] Petr Horava. Quantum Gravity at a Lifshitz Point. Phys. Rev., D79:084008, 2009, 0901.3775.

[33] Petr Horava. Membranes at Quantum Criticality. JHEP, 03:020, 2009, 0812.4287.

[34] Petr Horava. Spectral Dimension of the Universe in Quantum Gravity at a Lifshitz Point. Phys. Rev. Lett., 102:161301, 2009, 0902.3657.

[35] H. Lu, Jianwei Mei, and C. N. Pope. Solutions to Horava Gravity. Phys. Rev. Lett., 103:091301, 2009, 0904.1595.

[36] R.G. Cai, L.M. Cao, and N. Ohta. Topological black holes in Hořava-Lifshitz gravity. Physical Review D, 80(2):24003, 2009.

[37] A. Kehagias and K. Sfetsos. The black hole and FRW geometries of non-relativistic gravity. Physics Letters B, 678(1):123-126, 2009.

[38] Ahmad Ghodsi and Ehsan Hatefi. Extremal rotating solutions in Horava Gravity. Phys.Rev., D81:044016, 2010, 0906.1237.

[39] R.G. Cai, L.M. Cao, and N. Ohta. Thermodynamics of black holes in Horava-Lifshitz gravity. Physics Letters B, 679(5):504-509, 2009.

[40] R. Biswas and S. Chakraborty. Geometry of the thermodynamics of the black holes in HoravaLifshitz gravity. General Relativity and Gravitation, 43:41, 2011.

[41] S.W. Wei, Y.X. Liu, Y.Q. Wang, and H. Guo. Thermodynamic Geometry of black hole in the deformed Horava-Lifshitz gravity. Arxiv preprint arXiv:1002.1550, 2010.

[42] Steven Carlip and S. Vaidya. Phase transitions and critical behavior for charged black holes. Class.Quant.Grav., 20:3827-3838, 2003, gr-qc/0306054.

[43] Rabin Banerjee, Sumit Ghosh, and Dibakar Roychowdhury. New type of phase transition in Reissner Nordstróm - AdS black hole and its thermodynamic geometry. Phy. Lett. B, 696:156, 2011.

[44] Y.S. Myung. Thermodynamics of black holes in the deformed Horava-Lifshitz gravity. Physics Letters B, 678(1):127-130, 2009.

[45] Y.S. Myung. Entropy of black holes in the deformed Horava-Lifshitz gravity. Physics Letters $B, 684(2-3): 158-161,2010$.

[46] A. Castillo and A. Larranaga. Entropy for black holes in the deformed Horava-Lifshitz gravity. arXiv, 0906.4380.

[47] Y.S. Myung. ADM mass and quasilocal energy of black hole in the deformed Horava-Lifshitz 
gravity. Physics Letters B, 685(4-5):318-324, 2010.

[48] M. Wang, J. Jing, C. Ding, and S. Chen. First laws of thermodynamics in IR Modified Horava-Lifshitz gravity. Phys. Lett. B, 695:401, 2011.

[49] Mengjie Wang Songbai Chen Jiliang Jing. Second-order phase transition of Kehagias-Sfetsos black hole in deformed Hǒrava-Lifshitz gravity. Phys. Lett., B695:401, 2010, 1012.0645. 